\title{
Characterizations of NWP, ETGR and TWL Distributions
}

G.G. Hamedani

Department of Mathematics, Statistics and Computer Science

Marquette University

Milwaukee, WI 53201-1881, USA

g.hamedani@mu.edu

\begin{abstract}
Utilizing a simple relationship between two truncated moments as well as certain functions of the $1 s t$ and of the $n t h$ order statistics, we characterize three extended classes of distributions proposed in (2015).
\end{abstract}

Keywords: New Weibull-Pareto distribution; exponentiated transmuted generalized Rayleigh distribution; transmuted Weibull Lomax distribution; characterizations.

\section{Introduction}

The recent literature has suggested several ways of extending well know distributions. In a general way, generalized distributions provide a flexible framework for modeling a large range of data, that is, these models provide a rather flexible mechanism for fitting a wide spectrum of real world lifetime data in biology, medicine, engineering, economics, sports and other areas. In what follows we consider three generalized families of distributions introduced in 2015.

1) Nasiru and Luguterah (2015) proposed a New Weibull-Pareto (NWP) family of distributions with probability density function (pdf) given (in their own notation) by

$$
f_{N W P}(x)=f_{N W P}(x ; \beta, \delta, \theta)=\frac{\beta \delta}{\theta}\left(\frac{x}{\theta}\right)^{\beta-1} e^{-\delta\left(\frac{x}{\theta}\right)^{\beta}}, \quad x>0,
$$

and cumulative distribution function (cdf) in the form

$$
F_{N W P}(x)=F_{N W P}(x ; \beta, \delta, \theta)=1-e^{-\delta\left(\frac{x}{\theta}\right)^{\beta}}, \quad x \geq 0,
$$

where $\beta, \theta, \delta$ are all positive parameters.

2) Afify et al. (2015a) introduced the Exponentiated Transmuted Generalized Rayleigh (ETGR) family of distributions. The pdf of the ETGR family (in their own notation) takes the form $(x>0)$

$$
\begin{gathered}
f_{\text {ETGR }}(x)=f_{\text {ETGR }}(x ; \alpha, \beta, \lambda, \delta)=2 \alpha \delta \beta^{2} x e^{-(\beta x)^{2}}\left[1-e^{-(\beta x)^{2}}\right]^{\alpha \delta-1} \times \\
\left\{1+\lambda-2 \lambda\left[1-e^{-(\beta x)^{2}}\right]^{\alpha}\right\}\left\{1+\lambda-\lambda\left[1-e^{-(\beta x)^{2}}\right]^{\alpha}\right\}^{\delta-1},
\end{gathered}
$$

whereas the cdf is given by $(x \geq 0)$

$$
\begin{aligned}
& F_{E T G R}(x)=F_{E T G R}(x ; \alpha, \beta, \lambda, \delta) \\
& =\left[1-e^{-(\beta x)^{2}}\right]^{\alpha \delta} \times\left\{1+\lambda-\lambda\left[1-e^{-(\beta x)^{2}}\right]^{\alpha}\right\}^{\delta},
\end{aligned}
$$

where $\alpha, \beta, \delta$ all positive and $|\lambda| \leq 1$ are parameters. 
3) Afify et al. (2015b) proposed a new family of distributions called the Transmuted Weibull Lomax (TWL) family of distributions. The pdf and cdf of the TWL family (in their own notation) are given, respectively, by $(x>0)$

$$
\begin{aligned}
f_{T W L}(x)=f_{T W L} & (x ; \alpha, \beta, \lambda, a, b) \\
= & \frac{a b \alpha}{\beta}\left(1+\frac{x}{\beta}\right)^{b \alpha-1}\left[1-\left(1+\frac{x}{\beta}\right)^{-\alpha}\right]^{b-1} \\
& \times \exp \left\{-a\left[\left(1+\frac{x}{\beta}\right)^{\alpha}-1\right]^{b}\right\} \\
& \times\left\{(1+\lambda)-2 \lambda\left[1-\exp \left\{-a\left[\left(1+\frac{x}{\beta}\right)^{\alpha}-1\right]^{b}\right\}\right]\right\},
\end{aligned}
$$

and

$$
\begin{aligned}
F_{T W L}(x)=F_{T W L} & (x ; \alpha, \beta, \lambda, a, b) \\
& =\left[1-\exp \left\{-a\left[\left(1+\frac{x}{\beta}\right)^{\alpha}-1\right]^{b}\right\}\right] \\
& \times\left\{(1+\lambda)-\lambda\left[1-\exp \left\{-a\left[\left(1+\frac{x}{\beta}\right)^{\alpha}-1\right]^{b}\right\}\right],\right.
\end{aligned}
$$

where $\alpha, \beta, a, b$, all positive and $|\lambda| \leq 1$, are parameters.

It is widely known that the problem of characterizing a distribution is an important issue which has attracted the attention of many researchers. Thus, various characterizations have been established in many different directions. For example, we can refer to Galambos and Kotz (1978), Glänzel et al.(1984), Glänzel (1987, 1988, 1990), Hamedani (1993, 2002, 2006), Glänzel and Hamedani (2001), Bairamov et al. (2005), Ahsanullah and Hamedani (2007), Tavangar and Asadi (2007), Beg and Ahsanullah (2007), Bieniek (2007), Baratpour et al. (2007, 2008), Nevzotov et al. (2007), Su et al. (2008), Ahmadi and Fashandi (2009), Haque et al. (2009), Akhundov and Nevzorov (2010), Khan et al. (2010), Hamedani and Ahsanullah (2011), Yanev and Ahsanullah (2012), among others. The goal of this note is to provide characterizations of the NWP, ETGR and TWL families of distributions described above. These characterizations are based on: $(i)$ a simple relationship between two truncated moments, ( $i i)$ certain functions of the nth order statistic, ( $i i i)$ certain functions of the $1^{\text {st }}$ order statistic.

Although in many applications an increase in the number of parameters provides a more suitable model, in characterization problems a lower number of parameters (without seriously affecting the suitability of the model) is mathematically more appealing (see Glänzel and Hamedani 2001). In the applications where the underlying distribution is assumed to be NWP or ETGR or TWL distribution, the investigator needs to verify that the underlying distribution is in fact the NWP or ETGR or TWL distribution. To this end the investigator has to rely on the characterizations of these distributions and determine if the corresponding conditions are satisfied. Thus, the problem of characterizing these families of distributions become essential. As we mentioned earlier, our objective here is to present characterizations of the NWP, ETGR and TWL families of distributions. We shall do this in three different directions as discussed in Section 2 below. 


\section{Characterization Results}

The NWP, ETGR and TWL classes of distributions provide tools to obtain new parametric distributions from existing ones and have applications in many fields of study, in particular in lifetime modeling. So, an investigator will be vitally interested to know if their model fits the requirements of NWP or ETGR or TWL distribution. To this end the investigator riles on characterizations of these distributions, which provide conditions under which the underlying distribution is indeed a NWPor ETGR or TWL distribution. In this section we will present various characterizations of these distributions. First, we will consider characterizations based on two truncated moments. Next, characterizations based on truncated moments of certain functions of the $n t h$ order statistic and after that based on the 1 st order statistic.

\subsection{Characterizations based on two truncated moments}

In this subsection we present characterizations of the NWP, ETGR and TWL families of distributions in terms of a simple relationship between two truncated moments. The results derived here will employ an interesting theorem due to Glänzel (1987), which is given below.

Theorem 1. Let $(\Omega,, \mathbf{P})$ be a given probability space and let $H=[a, b]$ be an interval for some $a<b \quad(a=-\infty, b=\infty$ mightaswellbeallowed). Let $X: \Omega \rightarrow H$ be a continuous random variable with the distribution function $F$ and let $g$ and $h$ be two real functions defined on $H$ such that

$$
\mathbf{E}[g(X) \mid X \geq x]=\mathbf{E}[h(X) \mid X \geq x] \eta(x), \quad x \in H,
$$

is defined with some real function $\eta$. Assume that $g, h \in C^{1}(H), \eta \in C^{2}(H)$ and $F$ is twice continuously differentiable and strictly monotone function on the set $H$. Finally, assume that the equation $h \eta=g$ has no real solution in the interior of $H$. Then $F$ is uniquely determined by the functions $g, h$ and $\eta$, particularly

$$
F(x)=\int_{a}^{x} C\left|\frac{\eta^{\prime}(u)}{\eta(u) h(u)-g(u)}\right| \exp (-s(u)) d u,
$$

where the function $s$ is a solution of the differential equation $s^{\prime}=\frac{\eta^{\prime} h}{\eta h-g}$ and $C$ is a constant, chosen to make $\int_{H} d F=1$.

Remarks 1. (a) In Theorem G, the interval $H$ need not be closed. (b) The goal is to have the function $\eta$ as simple as possible. (c) It is possible to state Theorem 1 based on two functions $g$ and $\eta$ by setting $h(x) \equiv 1$ as we intend to do in the following Proposition.

Proposition 1. Let $X: \Omega \rightarrow(0, \infty)$ be a continuous random variable and let $h(x) \equiv 1$ and $g(x)=e^{-\delta\left(\frac{x}{\theta}\right)^{\beta}}$, for $x \in(0, \infty)$. The pdf of $X$ is (1) if and only if the function $\eta$ defined in Theorem 1 has the form

$$
\eta(x)=\frac{1}{2} e^{-\delta\left(\frac{x}{\theta}\right)^{\beta}}, \quad x>0 .
$$


Proof. Let $X$ have pdf (1), then

$$
(1-F(x)) \mathbf{E}[h(X) \mid X \geq x]=e^{-\delta\left(\frac{x}{\theta}\right)^{\beta}}
$$

and

$$
(1-F(x)) \mathbf{E}[g(X) \mid X \geq x]=\frac{1}{2} e^{-2 \delta\left(\frac{x}{\theta}\right)^{\beta}},
$$

and finally

$$
\eta(x) h(x)-g(x)=\eta(x)-g(x)=-\frac{1}{2} e^{-\delta\left(\frac{x}{\theta}\right)^{\beta}}<0, \quad x>0 .
$$

Conversely, if $\eta$ is given as above, then

$$
s^{\prime}(x)=\frac{\eta^{\prime}(x) h(x)}{\eta(x) h(x)-g(x)}=\frac{\eta^{\prime}(x)}{\eta(x)-g(x)}=\frac{\beta \delta}{\theta}\left(\frac{x}{\theta}\right)^{\beta-1}, x>0,
$$

and hence

$$
s(x)=\delta\left(\frac{x}{\theta}\right)^{\beta}, \quad x>0
$$

Now, in view of Theorem $1, X$ has cdf (2) and pdf (1).

Corollary 1. Let $X: \Omega \rightarrow(0, \infty)$ be a continuous random variable and let $h(x)$ be as in Proposition 1. The pdf of $X$ is (1) if and only if there exist functions $g$ and $\eta$ defined in Theorem 1 satisfying the differential equation

$$
\frac{\eta^{\prime}(x)}{\eta(x)-g(x)}=\frac{\beta \delta}{\theta}\left(\frac{x}{\theta}\right)^{\beta-1}, \quad x>0 \text {. }
$$

Remarks 2. (a) The general solution of the differential equation in Corollary 1 is

$$
\eta(x)=e^{\delta\left(\frac{x}{\theta}\right)^{\beta}}\left[-\int \frac{\beta \delta}{\theta}\left(\frac{x}{\theta}\right)^{\beta-1} e^{-\delta\left(\frac{x}{\theta}\right)^{\beta}} g(x) d x+D\right], \quad x>0,
$$

where $D$ is a constant. One set of appropriate functions is given in Proposition 1 with $D=0$.

(b) Clearly there are other triplets of functions $(h, g, \eta)$ satisfying the conditions of Theorem 1. We presented one such triplet in Proposition 1.

The proofs of the following two Propositions are similar to that of Proposition 1 and hence will be omitted.

Proposition 2. Let $X: \Omega \rightarrow(0, \infty)$ be a continuous random variable and let $h(x)=$ $\left\{1+\lambda-2 \lambda\left[1-e^{-(\beta x)^{2}}\right]^{\alpha}\right\}^{-1}\left\{1+\lambda-\lambda\left[1-e^{-(\beta x)^{2}}\right]^{\alpha}\right\}^{1-\delta}$ and $\quad g(x)=h(x)[1-$ 
$\left.e^{-(\beta x)^{2}}\right]^{\alpha \delta}$ for $x \in(0, \infty)$. The pdf of $X$ is (3) if and only if the function $\eta$ defined in Theorem 1 has the form

$$
\eta(x)=\frac{1}{2}\left\{1+\left[1-e^{-(\beta x)^{2}}\right]^{\alpha \delta}\right\}, \quad x>0 .
$$

Proposition 3. Let $X: \Omega \rightarrow(0, \infty)$ be a continuous random variable and let $h(x)=$ $\left\{(1+\lambda)-2 \lambda\left[1-\exp \left\{-a\left[\left(1+\frac{x}{\beta}\right)^{\alpha}-1\right]^{b}\right\}\right]\right\}^{-1}$ and $g(x)=h(x) \exp \left\{-\left[\left(1+\frac{x}{\beta}\right)^{\alpha}-\right.\right.$ $\left.1]^{b}\right\}$ for $x \in(0, \infty)$. The pdf of $X$ is (5) if and only if the function $\eta$ defined in Theorem 1 has the form

$$
\eta(x)=\frac{a}{a+1} \exp \left\{-\left[\left(1+\frac{x}{\beta}\right)^{\alpha}-1\right]^{b}\right\}, \quad x>0 .
$$

\subsection{Characterizations based on truncated moment of the $n t h$ order statistic}

Let $X_{1: n} \leq X_{2: n} \leq \ldots \leq X_{n: n}$ be the corresponding order statistics from a random sample of size $n$ of a continuous cdf $F$. We briefly discuss here two characterization results based on functions of the $n t h$ order statistic. We have the following proposition.

Proposition 4. Let $X: \Omega \rightarrow(0, \infty)$ be a continuous random variable with cdf $F$. Let $\psi$ and $q$ be two differentiable functions on $(0, \infty)$ such that

$$
\lim _{x \rightarrow 0^{+}} \psi(x)[F(x)]^{n}=0, \quad \int_{0}^{\infty} \frac{q^{\prime}(t)}{[\psi(t)-q(t)]} d t=\infty .
$$

Then

$$
E\left[\psi\left(X_{n: n}\right) \mid X_{n: n}<t\right]=q(t), \quad t>0,
$$

implies

$$
F(x)=\exp \left\{-\int_{x}^{\infty} \frac{q^{\prime}(t)}{n[\psi(t)-q(t)]} d t\right\}, \quad x \geq 0 .
$$

Proof. If (7) holds, then using integration by parts on the left hand side of (7) and the assumption $\lim _{x \rightarrow 0^{+}} \psi(x)[F(x)]^{n}=0$, we have

$$
\int_{0}^{t} \psi^{\prime}(x)(F(x))^{n} d x=[\psi(t)-q(t)](F(t))^{n} .
$$

Differentiating both sides of the above equation with respect to $t$, we arrive at

$$
\frac{f(t)}{F(t)}=\frac{q^{\prime}(t)}{n[\psi(t)-q(t)]}, \quad t>0
$$

Now, integrating (9) from $x$ to $\infty$, we have, in view of $\int_{0}^{\infty} \frac{q^{\prime}(t)}{[\psi(t)-q(t)]} d t=\infty$, a cdf $F$ given by (8). 
Remarks 3. (a) Taking, for instance, $\psi(x)=2\left[\left\{1-e^{-(\beta x)^{2}}\right\}^{\alpha}\{1+\lambda-\lambda[1-\right.$ $\left.\left.\left.e^{-(\beta x)^{2}}\right]^{\alpha}\right\}\right]^{n \delta}$ and $q(x)=\frac{1}{2} \psi(x)$ in Proposition 4 , from equation (8) we will have a cdf $F$ given by (4). (b) Taking, for instance, $\psi(x)=2\left\{\left[1-\exp \left\{-a\left[\left(1+\frac{x}{\beta}\right)^{\alpha}-\right.\right.\right.\right.$ $\left.\left.\left.1]^{b}\right\}\right]\left\{(1+\lambda)-\lambda\left[1-\exp \left\{-a\left[\left(1+\frac{x}{\beta}\right)^{\alpha}-1\right]^{b}\right\}\right]\right\}\right\}^{n} \quad$ and $\quad q(x)=\frac{1}{2} \psi(x) \quad$ in Proposition 4, from equation (8) we will have a cdf $F$ given by (6). (c) Clearly there are other pairs of functions $(\psi, q)$ which satisfy conditions of Proposition 4.

\section{Characterizations based on truncated moment of the $\mathbf{1}^{\text {st }}$ order statistic}

We state here a characterization base on certain functions of the $1^{\text {st }}$ order statistic. We like to mention here that the proof of Proposition 5 below is straightforward extension of that of Theorem 2.2 of Hamedani (2010). We give a short proof of it here for the sake of completeness.

Proposition 5. Let $X: \Omega \rightarrow(0, \infty)$ be a continuous random variable with cdf $F$. Let $\psi(x)$ and $q(x)$ be two differentiable functions on $(0, \infty)$ such that

$$
\lim _{x \rightarrow \infty} \psi(x)[1-F(x)]^{n}=0, \quad \int_{0}^{\infty} \frac{q^{\prime}(t)}{[q(t)-\psi(t)]} d t=\infty .
$$

Then

$$
E\left[\psi\left(X_{1: n}\right) \mid X_{1: n}>t\right]=q(t), \quad t>0,
$$

implies

$$
F(x)=1-\exp \left\{-\int_{0}^{x} \frac{q^{\prime}(t)}{n[q(t)-\psi(t)]} d t\right\}, \quad x \geq 0 .
$$

Proof. If (10) holds, then using integration by parts on the left hand side of (10) and the assumption $\lim _{x \rightarrow \infty} \psi(x)[1-F(x)]^{n}=0$, we have

$$
\int_{t}^{\infty} \psi^{\prime}(x)(1-F(x))^{n} d x=[q(t)-\psi(t)](1-F(t))^{n} .
$$

Differentiating both sides of the above equation with respect to $t$, we arrive at

$$
\frac{f(t)}{1-F(t)}=\frac{q^{\prime}(t)}{n[q(t)-\psi(t)]}, \quad t>0
$$

Now, integrating (12) from 0 to $x$, we have, in view of $\int_{0}^{\infty} \frac{q^{\prime}(t)}{[q(t)-\psi(t)]} d t=\infty$, a cdf $F$ given by (11). 
Remark 4. Taking, for instance, $\psi(x)=2 n \delta\left(\frac{x}{\theta}\right)^{\beta}$ and $q(x)=\frac{1}{2} \psi(x)$ in Proposition 5 , from equation (11) we will have a cdf $F$ given by (2).

\section{References}

1. Afify, A.Z., Nofal, Z.M. and Ebraheim, A.E.H.N. (2015a). Exponentiated transmuted generalized Rayleigh distribution: A new four parameter Rayleigh distribution. Pak.j.stat.oper.res., XI, 115-134.

2. Afify, A.Z., Nofal, Z.M. and Yousof, H.M. (2015b). The transmuted Weibull Lomax distribution: Properties and Application. Pak.j.stat.oper.res., XI, 135-152.

3. Ahmadi, J., Fashandi, M. (2009). Some characterization and ordering results on entropies of current records. Statistics and Probability Letters, 79, 2053-2059.

4. Akhundov, I., Nevzorov, V.B. (2010). A simple characterization of Student's $t_{3}$ distribution. Statistics and Probability Letters, 80, 293-295.

5. Ahsanullah, M., Hamedani, G.G. (2007). Certain characterizations of power function and beta distributions based on order statistics. Journal of Statistical Theory and Applications, 6, 220-226.

6. Bairamov, I., Ahsanullah, M., Pakes, A.G. (2005). A characterization of continuous distributions via regression on pairs of record values. Australian and New Zealand Journal of Statistics, 47, 543-547.

7. Baratpour, S., Ahmadi, J., Arghami, N.R. (2007). Some characterizations based on entropy of order statistics and record values. Communications in StatisticsTheory and Methods, 36, 47-57.

8. Baratpour, S., Ahmadi, J., Arghami, N.R. (2008). Characterizations based on Renyi entropy of order statistics and record values. Journal of Statistical Planning and Inference, 138, 2544-2551.

9. Beg, M.I., Ahsanullah, M. (2007). On characterizing distributions by conditional expectations of functions of generalized order statistics. Journal of Applied Statistical Science, 15, 229-244.

10. Bieniek, M. (2007). On characterizations of distributions by regression of adjacent generalized order statistics. Metrika, 66, 233-242.

11. Galambos, J. and Kotz, S. (1978). Characterizations of probability distributions. A unified approach with an emphasis on exponential and related models, Lecture Notes in Mathematics, 675, Springer, Berlin.

12. Glänzel, W. (1987). A characterization theorem based on truncated moments and its application to some distribution families. In Mathematical Statistics and Probability Theory (Bad Tatzmannsdorf, 1986), Vol. B (p. 75-84). Dordrecht: Reidel.

13. Glänzel, W. (1988). A characterization of the normal distribution. Studia Scientiarum Mathematicarum Hungarica, 23, 89-91. 
14. Glänzel, W. (1990). Some consequences of a characterization theorem based on truncated moments, Statistics, 21, 613-618.

15. Glänzel, W., Hamedani, G.G. (2001). Characterizations of univariate continuous distributions. Studia Scientiarum Mathematicarum Hungarica, 37, 83-118.

16. Glänzel, W., Telcs, A., Schubert, A. (1984). Characterization by truncated moments and its application to Pearson-type distributions. Zeitschrift für Wahrscheinlichkeitshéorie und Verwandte Gebiete, 66, 173-183.

17. Hamedani, G.G. (1993). Characterizations of Cauchy, normal and uniform distributions. Studia Scientiarum Mathematicarum Hungarica, 28, 243-247.

18. Hamedani, G.G. (2002). Characterizations of univariate continuous distributions. II. Studia Scientiarum Mathematicarum Hungarica, 39, 407-424.

19. Hamedani, G.G. (2006). Characterizations of univariate continuous distributions. III. Studia Scientiarum Mathematicarum Hungarica, 43, 361-385.

20. Hamedani, G.G. (2010). Characterizations of univariate continuous distributions based on truncated moments of functions of order statistics. Studia Scientiarum Mathematicarum Hungarica, 47, 462-468.

21. Hamedani, G.G., Ahsanullah, M. (2011).

22. Haque, Z., Athar, H., Khan, R.U. (2009). Characterization of probablity distributions through expectation of function of generalized order statistics. Journal of Statistical Theory and Applications, 8, 416-426.

23. Khan, A.H., Anwar, Z., Chishti, S. (2010). Characterization of continuous distributions through conditional expectation of functions of dual generalized order statistics. Pakistan Journal of Statistical, 26, 615-628.

24. Nasiru, S. and Luguterah, A. (2015). The new Weibull-Pareto distribution. Pak.j.stat.oper.res., XI, 103-114.

25. Nevzorova, L., Nevzorov, I., Akhundov, I. (2007). A simple characterization of Student's $t_{2}$ distribution. Metron, LXV, 53-57.

26. Su, J.C., Su, N.C., Huang, W.J. (2008). Characterizations based on record and order statistics. Journal of Statistical Planning and Inference, 138, 1358-1367.

27. Tavangar, M., Asadi, M. (2007). Generalized Pareto distributions characterized by generalized order statistics. Communications in Statistics-Theory and Methods, 36, 1333-1341.

28. Yanev, G.P., Ahsanullah, M. (2012). Characterizations of Student's t-distribution via regressions of order statistics. Statistics, 46, 429-435. 